\title{
Tangence
}

\section{Écrire, habiter}

\section{Alain Masson}

Numéro 58, octobre 1998

Le postmoderne acadien

URI : https://id.erudit.org/iderudit/025978ar

DOI : https://doi.org/10.7202/025978ar

Aller au sommaire du numéro

Éditeur(s)

Tangence

ISSN

0226-9554 (imprimé)

1710-0305 (numérique)

Découvrir la revue

Citer cet article

Masson, A. (1998). Écrire, habiter. Tangence, (58), 35-46.

https://doi.org/10.7202/025978ar

Ce document est protégé par la loi sur le droit d'auteur. L'utilisation des services d'Érudit (y compris la reproduction) est assujettie à sa politique d'utilisation que vous pouvez consulter en ligne.

https://apropos.erudit.org/fr/usagers/politique-dutilisation/
Cet article est diffusé et préservé par Érudit.

Érudit est un consortium interuniversitaire sans but lucratif composé de l'Université de Montréal, l'Université Laval et l'Université du Québec à Montréal. Il a pour mission la promotion et la valorisation de la recherche. https://www.erudit.org/fr/ 


\section{Écrire, habiter}

\section{Alain Masson}

Écrire et habiter, c'est occuper un peu d'espace, sur la page, dans le monde. Au mythe originel de la dispersion des Acadiens répond la rareté des signes sur les feuilles blanches. France Daigle bien sûr, mais aussi cette littérature dominée par la poésie, écriture timide qui n'emplit pas la ligne, et chez Gérald Leblanc, le goût de la brisure. Mais aussi la fascination des fragments, qui semble priver certains écrivains de la continuité nécessaire au roman: Jean Babineau, par exemple.

\section{Le locataire de l'étendue}

J'écris au feutre

sur une carte de l'Amérique ${ }^{1}$

Simples et lucides, ces deux vers de Gérald Leblanc marquent l'ambition et les limites de son projet. Impatient de s'approprier les lieux, le poète acadien n'entreprend pas, à l'imitation de ses aînés québécois, de "nommer le pays", il en conteste la représentation. Sil lance un geste possessif,

comme un lasso

autour du territoire

(CC, p. 12)

ce n'est que durant le temps du poème. Les villes seront des "refuges temporaires" où "nos mots s'immiscent autour d'un autre départ. ( $C C$, p. 14). La nomination du lieu, dans ce beau pentamètre iambique puis anapestique (c'est le rythme d'un pas qui gagne en ampleur), demeure clandestine et ne se sépare pas d'une partance. Habiter, c'est tenir un monde autour de soi comme le sien. Or Gérald Leblanc ne détient qu'une parole. Il écrit souvent "ma rue", "ma ville", mais ces possessifs ne

1 Gérald Leblanc, Complaintes du continent, poèmes 1988-1992, Moncton/ Trois-Rivières, Éditions Perce-Neige/Écrits des Forges, 1993, p. 13. Dorénavant, les renvois à cet ouvrage seront identifiés par le sigle $C C$, suivi du folio 
sauraient faire illusion: "la rue est une idée" ${ }^{2}$, note Eloge $d u$ chiac, c'est "la rue de la ruse d'être là " (ÉC, p. 58). Quant à Moncton, on sait depuis les Complaintes que c'est une "villelangues" (CC, p. 24) et

il est des mots

dont la seule mention éveille

la certitude d'habiter un espace

qui répond au sens de ces mots

(CC, p. 19)

Quelle précision! À l'acte souverain de la nomination répond la simple mention: l'énoncé autonymique où le vocable se désigne sans pouvoir rien désigner d'autre; sans doute, c'est le moyen de souligner la valeur du mot; dans le poème aussi, il appelle l'attention sur lui-même; mais peut-il faire mieux? Au thème du pays s'oppose l'espace le moins défini, qui n'ouvre qu'une valeur verbale. Ainsi le monde habitable ne se constitue que dans l'acte poétique lui-même. On n'habite qu'en écrivant. Las d'habiter à Moncton, d'écrire à Moncton, Gérald Leblanc, écrivant Moncton, habitera Moncton.

La ville en effet se laisse surtout pénétrer comme une essence purement sonore. Complaintes $d u$ continent : "je me love dans les bruits de la ville. ( $C C, \mathrm{p} .21)$. C'est l'acte fondateur. Poète urbain, Gérald Leblanc ne s'imagine ni comme un Tityre à l'aise sous son hêtre, ni comme un écrivain studieux à sa table de travail. S'il évoque sa demeure, c'est comme un désordre. La composition semble requérir une déambulation, voisine de l'errance de Bloupe, mais heureuse. Habiter la maison brûlée de France Daigle ou, "glorieusement" mais "avec beaucoup de jeux de mots" (ÉC, p. 116), les rues, c'est la revendication d'Éloge du chiac. Dire que "nous habitons une langue" ( $C C$, p. 25), ce n'est pas seulement élever, la métaphore serait banale, le français au rang d'une demeure, c'est requérir de la parole qu'elle prenne toute la place de l'habitation. Par un retournement hardi, le double signe de la frustration acadienne, le bilinguisme imposé et le territoire refusé, se change en une fondation héroïque. Voilà le mouvement décisif des Complaintes du continent. N'ayant pour lui ni l'évidence d'une langue ni la possession d'un domaine, le poète transforme

2 Gérald Leblanc, Éloge du chiac, Moncton, Éditions Perce-Neige, 1995, p. 40. Dorénavant, les renvois à cet ouvrage seront identifiés par le sigle $E C$, suivi du folio. 
cette double négativité en synonymie habitable. C'est ainsi que le dépossédé entre en jouissance. La langue est sa maison, puisque ni la langue ni la maison ne lui appartiennent. La proposition est assurément un sophisme, le mouvement de la pensée poétique établit une vérité qui n'appartient qu'à elle, mais qui comble une attente. Elle ne constate pas, elle engage: "ma vie s'appelle Moncton" ( $C C$, p. 80$)$.

Peut-on demander moins? peut-on faire plus? "Fill in the blanks" ( $C C$, p. 28), telle est la devise: "j'écris la distance" ( $C C$, p. 28). L'écriture n'exprime pas une nation, elle institue une façon, patriotique à sa manière, de vivre sans nation. Mais l'habitation du continent, au terme de cet essor, compensera l'inaccessibilité du territoire: «j'habite l'Amérique" (CC, p. 77), une Amérique non spatialisée, qui s'exprime inlassablement dans la circulation des sons et des signes. L'intensité est la marque qui distingue ce paysage intime et immense et qui, depuis toujours, en rend la géographie perceptible au poète. La "cartographie acoustique ", "l'oreille au laser ( $C C$, p. 80) accomplissent pour finir la tentative un peu maladroite d'imposer des noms à la carte. Un élément plus secret, plus délicat, plus inconstant, mais un élément où vivre, se substitue à l'espace que le poète soumettait d'abord à la question. Il ouvre toute l'étendue, présente dans la parole. Reprenant le cri initial de Raymond LeBlanc, Gérald Leblanc en modifie le sens d'une manière décisive: en ajoutant au "cri de terre", si désespérément épris de la nostalgie d'un pays, son "cri de fer" et son "cri de mer" (CC, p. 61), il change la valeur du complément de nom; celui-ci ne peut plus désigner ni le but ni la cause (je crie pour une terre; la terre crie par ma bouche), il note la qualité ou la matière du cri, ce qui suppose l'absorption par la parole de la terre, de la mer, du fer (mon cri est fait de fer, de terre, de mer). Cette métamorphose syntaxique d'une citation appartient bien au génie contemporain : la connaissance attentive du passé (le poème s'intitule "hommage" [CC, p. 61]) ne va pas sans une déformation subtile, dans le sens d'une subjectivité accentuée et d'une confiance, peut-être excessive, dans la puissance de l'énonciation.

Postmoderne? Sans doute pas: le patriotisme de la langue demeure attachement à une tradition vivante, et récuse le jeu avec des formes anciennes. Voici un point crucial: cet attachement n'apparaît nullement comme une identité, encore moins comme un enracinement; il s'exerce dans la mobilité, il consent à l'usage 
38

de l'américain et de sa culture, mais aussi du sanskrit; il n'emprunte guère au parler acadien; Complaintes du continent est tout à fait exempt de chiac. Les idiomes locaux ne désignent que des phénomènes proprement régionaux: les "flatteux de bedaines "( $C C$, p. 79), le "big business" (CC, p. 77), "weird" (CC, p. 70), voilà des expressions qui perdraient toute valeur à être traduites. Mais la langue du recueil est le français littéraire, qu'il faudrait bien que les Français cessassent de considérer comme leur apanage. Ce choix peut paraître étrange de la part d'un poète épris de musique et habitant de l'intensité sonore de l'Amérique. Pourtant, l'Éloge du chiac le confirme:

nous emporterons dans la langue

les mots ramassés en chemin

(ÉC, p. 11)

Gérald Leblanc renonce tout à fait à l'imitation directe de l'oralité. La langue, en effet, suppose plus que les rencontres de termes, heureuses ou non, en chemin; elle entraîne une esthétique. Lorsqu'il écrit en américain, le poète conserve une diction distinctement acadienne. S'il use d'un style sec, nourri de vocabulaire banal et de monosyllabes familiers, son rythme est moins syncopé, moins sensible au beat accentuel que celui des poètes de "l'autre langue". De surcroit, il emploie moins volontiers les ressources de la nominalisation, les mots composés, les épithètes multiples, l'ambiguiité catégorielle des formes lexicales, ressources caractéristiques de la poésie américaine contemporaine. Son idée de la clarté syntaxique demeure résolument française. De même, chez Jean Babineau, l'américain et le chiac ressortissent à un usage littéraire du français: officer ne peut devenir "ossifer " ${ }^{3}$ que dans une langue qui possède le nom os et le verbe ossifier. Nul particularisme chez nos trois écrivains.

"La musique provoque des perceptions aiguës." (CC, p. 53) Voilà, dans les Complaintes, un vers clair, presque banal. On écoutera seulement l'allitération en $/ \mathrm{p} /$, mais surtout la façon dont le rythme accentuel, dans la première partie du vers, isole deux $e$ muets, après deux anapestes: "coupe lyrique" qui ralentit et contrarie l'élan, en soulignant un son qu'on murmure à peine et qui devient l'objet d'une perception aiguë. Cet art, presque

3 Jean Babineau, Bloupe, Moncton, Éditions Perce-Neige, 1993, p. 152. Dorénavant, les renvois à cet ouvrage seront identifiés par le sigle $B$, suivi du folio. 
classique, transpose et évoque l'expérience du matériau sonore. Il ne prétend pas exprimer l'intensité naïvement, il en traduit sensuellement la forme, et elle seule.

Malgré son titre, Éloge du chiac n'use de ce parler qu'avec discrétion, avec une dilection voisine de la préciosité. L'observation d'une parataxe propre au langage parlé, dans une formule comme "je ne m'en inquiète guère, y a vraiment rien de wrong avec ça" (EC, p. 7), accentue le contraste entre l'archaïsme du premier membre de phrase et la tournure chiaque du second, de sorte que l'effet relève plutôt de l'esprit que de l'assujettissement à une expression populaire: "ça brasse dans le lexique, et en pleine modernité", c'est-à-dire sans concession à la coutume, sans épouser servilement une identité.

En outre, cette poésie demeure remarquablement sobre et grave. Elle a ses raisons. Elle ne cultive ni l'individualisme ni le romantisme révolutionnaire. Elle invite à vivre le monde dans une langue. C'est "une proposition de tolérance" (EC, p. 13), remarque l'Éloge du cbiac. On imagine mal une version plus modeste et plus sûre des fondements de l'Acadie. Comme par dessous le politique, ce qui ne l'exclut nullement. On peut la juger résolument contemporaine: elle dispose de son action et de ses progrès sans connaître ses principes. Postmoderne, non: elle n'entend nullement rompre avec une vaste entreprise constructive.

\section{L'inhabitante}

Les œuvres de France Daigle n'y renoncent pas davantage. Le protagoniste de La beauté de l'affaire ${ }^{4}$ est un architecte; son activité paraît sous un jour religieux, à la fois chrétien et maçonnique, puisqu'il prie le "Grand Bâtisseur" clans la "maison du Seigneur" ( $B A, \mathrm{p} .7$ ), pendant la messe de minuit. Il rêve de "maisons à construire qui soient plus parfaites encore" ( $B A, \mathrm{p}$. 7). Mais c'est le roman lui-même qui devient "jeu d'assemblage et de construction" ( $B A$, p. 13). L'argument du livre veut en effet que la voix du commentaire, d'abord solennelle et indépendante, puis rapportée à la troisième personne du singulier et au féminin

4 France Daigle, La beauté de laffaire, Moncton/Montréal, Éditions d'Acadie/Éditions NBJ, 1991, 60 p. Dorénavant, les renvois à cet ouvrage seront iclentifiés par le sigle $B A$, suivi du folio. 
40

(elle), ce qui l'assimile à l'épouse délaissée du constructeur, se distingue de plus en plus mal de la pensée de ce dernier. Car la pertinence du récit repose sur les critères des chantiers: un homme qui construit une clôture dans une île, un projet social d'établissement culturel. Les notations sur la construction, les métaphores qu'elle inspire se multiplient au fil du texte. Le volume se ferme sur le fac-similé d'une facture rose adressée à "Mrs France Daigle" par Eastern Fence Ltd (BA, p. 57); ce finale répond ironiquement à la citation de l'Évangile selon saint Jean: "Et le Verbe s'est fait chair, et il a babité parmi nous." (BA, p. 54) Ainsi l'œuvre de la parole, l'incarnation, la construction s'arrangent-elles en une signification complexe: bâtir apparaît comme une nécessité artistique et humaine, mais s'il n'est pas de construction sans clôture, toute œuvre éveille la nostalgie irrémédiable d'un "espace total" où la narratrice rêve, un peu comme Gérald Leblanc, d'être enfin "rendue" ( $B A$, p. 35), c'est-à-dire arrivée à destination, mais aussi restituée à elle-même, maîtresse de soi comme de son patrimoine. La chose est impossible. Habiter passe donc aux yeux de l'écrivain pour une incarnation privatrice. De là cette réticence à remplir la page comme le faisait Duras (il est vrai que celle-ci remplissait beaucoup). L'un des projets, d'ailleurs voué à l'échec, concerne un simple espace vert, mais fermé d'un grillage. "La barrière des mots, œuvre de clôture" (BA, p. 23) : la séparation, l'usage des "sentinelles" verbales, est inévitable dans l'espace du bilinguisme, mais elle est inévitablement malheureuse. L'écrivain donne ainsi une version plus critique de la grande opposition entre l'étendue et l'exiguité que Gérald Leblanc surmontait pour habiter l'Amérique.

Le hasard des idiomes veut qu'en nommant France Daigle l'inhabitante, on résumerait sa situation: la langue dominante la tient ainsi pour une habitante, la langue qu'elle parle reconnaîtrait par là qu'elle ne se résout pas à habiter.

C'était précisément le sujet d'Histoire de la maison qui brûle ${ }^{5}$. Les personnages sont figés, ce qui rend tout récit impossible: le narrateur est arrêté sur place par la vision d'une femme assise sur le trottoir d'en face, alors qu'il allait chercher à la poste des formulaires destinés à la préparation de colis pour Émile

5 France Daigle, Histoire de la maison qui brâle, Moncton, Éditions d'Acadie, 1985. Dorénavant, les renvois à cet ouvrage seront identifiés par le sigle $H M B$, suivi du folio. 
Lauvrière; par ailleurs une maison brûlait, sans doute celle qu'avait habitée la femme immobile, sans domicile fixe. Or Lauvrière, on le sait, est l'auteur de La tragédie d'un peuple ${ }^{6}$, qui évoque le Grand Dérangement. L'incendie rappelle explicitement celui de Grandpré "qui dévorait granges, maisons, églises" ( $H M B$, p. 49). Le narrateur se voit donc empêché d'accomplir une petite besogne littéraire, correspondre avec un écrivain porteur du passé national. Blocage: le temps devient un "symbole" ( $H M B$, p. 17) qui réunit cette rencontre de "novembre 1953" ( $H M B$, p. 16), le fait divers de "la maison qui brûle" ( $H M B$, p. 97) et les crimes de guerre perpétrés en 1755 par les Britanniques. Plutôt qu'une histoire, c'est cet assemblage qui constitue la fiction. L'appropriation de la tragédie historique, que justifie l'intercession lointaine de Lauvrière avec lequel le rapport, la correspondance, ne peut précisément s'établir, ouvre une figure vraiment romanesque, accommodant non sans retenue le grandiose et le banal. Mais l'espace n'est pas moins altéré que le temps. À la représentation géographique se substitue la présence d'un élément où voisinent "cette rue de Montréal " (HMB, p. 102), proche de la rue Union, et la "campagne" ou "la petite rue Doucet. ( $H M B$, p. 88), à Dieppe, où la maison brûle: un seul élan semble àvoir mené la femme d'un lieu à l'autre. De plus si l'on considère les stratégies narratives en termes de positions, le point de vue rapproche le narrateur de cette femme, dont il connaît toutes les émotions et dont il partage l'immobilité, en la voyant "au fond de moi, de l'autre côté de la rue" (HMB, p. 9): cette formule fondatrice repose-t-elle sur la sympathie ou sur le souvenir? Elle exprime en tout cas le principe du livre, typographie comprise: "Toujours la page de droite renvoyait à celle de gauche et on ne s'attendait plus à des livres épais aux pages bien remplies." ( $H M B$, p. 14) Paradoxe, peut-être: l'espacement abolit la distance, l'évidement rapproche. Faute d'un lieu topographiquement défini, la narration se déploie et rassemble. L'inhabitation est l'humanité même: ce que Rousseau appelait la "bienveillance" ou la "pitié ". L'institution qui permet la correspondance s'appelle donc "la maison de la poste" ( $H M B$, p. 32), ce qui ne paraît guère conforme à l'usage acadien; elle reste introuvable. Son rôle est proche de celui que tient "la maison du

36. Émile Lauvrière, La tragédie d'un peuple: bistoire du peuple acadien des origines à nos jours, Paris, Bossard, 1922. 
42

Seigneur "dans La beauté de l'affaire: il n'y a pas d'autre demeure que celle qui s'ouvre au rapport avec l'autre.

Les livres de France Daigle, écrits dans une langue de plus en plus sobre et de plus en plus nette, ne répondent sans doute guère à l'injonction des lecteurs et des critiques, qui veulent toujours que l'on raconte. Raconter quoi? Encore faudrait-il trouver une histoire inédite! Cependant la minutie de la construction et la liberté du montage n'ont pas pour matériau morphologique, comme chez Claude Simon, l'ambiguité des images: c'est une chorégraphie de personnages, lente et émouvante, rapprochements, distances, tâches ressemblantes. C'est une concomitance des âmes. Le rejet de l'ornement, le refus du foisonnement, l'évidence de la règle, s'ils répondent au goût contemporain, s'éloignent de l'éclectisme postmoderne. Le souci de l'espace n'a rien d'un jeu, ni d'une simple réflexion: l'écriture organise effectivement un monde, parce qu'elle propose, énigmatiques mais déchiffrables, des relations. Les personnages ne sont pas gouvernés par des mobiles, comme ceux des romans ordinaires; la fiction ne tranche pas entre leurs projets; indécis, immobiles, absorbés, ils se frôlent ou se côtoient. Mais des échanges impondérables, indéfinissables, les unissent.

\section{Sans laisser d'adresse.}

Dans Bloupe de Jean Babineau, la Poste a aussi sa place. Bloupe exerce assez longtemps les fonctions de facteur. Mais l'institution ne semble pas très efficace! La graphomanie du postier l'empêcherait plutôt de prendre du courrier le soin qu'il mérite. Cela devient les "Bostes" ( $B$, p. 34), le "bourrier" $(B$, p. 24). À la correspondance s'oppose une écriture individualiste, secrète, plus soucieuse de harceler les langues que de manifester des vérités. Écrire, c'est d'abord pour Bloupe un problème de position corporelle: il faut trouver un coin propice, il faut caser les manuscrits. Par là le thème de l'écriture entre en résonance avec deux autres thèmes récurrents du livre: les déplacements constants, souvent assortis d'accidents, de chutes, de maladresses, de pertes, d'erreurs, d'égarements; et les postures consternantes et burlesques que prend le protagoniste chez lui, dans son lieu de travail, dans une auto, dans sa cave, dans une boîte, pour lire, pour écrire, pour dormir, pour rien. Recherche désespérée d'un abri. Dans sa maison, Bloupe ne tient pas en 
place, s'assied sur le fridge, se couche par terre: la demeure est par excellence un lieu d'errance, une possibilité cle désordre infantile. On comprend vite que l'aspect peu maîtrisé du livre et de la vie dont il rend compte, sans chronologie ni intrigue, favorise l'expansion du moi littéraire, qui lasse parfois le lecteur. Mais c'est une rencontre intéressante qui associe ce jaillissement, d'ailleurs discontinu, à l'inconfort, à l'instabilité, à l'étroitesse, à l'exiguité du temps ("j'ai vingt minutes pour écrire une page de mots" $[B$, p. 98]) et de l'espace ("écrire un texte sur le capot de l'auto" $[B$, p. 54] $)$, à la contrainte physique. C'est du même coup que Bloupe s'étrangle et s'étale. La multiplication des niveaux est le ressort de la machine: Bloupe passe d'une langue à l'autre, de l'usage littéraire au chiac ou au slang, d'un point de vue à l'autre, de la citation au pastiche, d'une voix à l'autre, de l'auto-citation au récit, du soliloque polyglotte à la notation précise, du journal au monologue intérieur, sans crier gare. L'écriture vivante se laisse quelquefois interrompre par l'écriture citée, qui comporte elle aussi au moins deux degrés: la pensée écrivante et le soliloque qui la commente. Ainsi l'écriture recèle du temps tout en s'appropriant le temps, en un travail toujours débordé. L'échelonnement imprévisible des formes compose un espace qui s'approfondit, "un autre pays de pages" ( $B$, p. 96$)$, mais c'est un horizon qui se dérobe. Écrire est pour Bloupe un verbe intransitif, qui n'admet que le complément d'objet interne: il écrit des mots, des pages, des textes, non une vie ou un roman. C'est la "théorie de la curve ball" ( $B$, p. 89). Imprévisible pour le batteur, pour le lecteur, pour l'oppresseur, mais aussi pour le lanceur; pour l'écrivain, "flottante", voilà la prose. Pourquoi? La réponse est nette, page 89: "pas de pays". C'est tout le contraire de la théorie de la toupie, exposée par France Daigle dans Histoire de la maison qui brûle: "les toupies finissent toujours par trouver leur équilibre et leur axe malgré des vacillements initiaux" (HMB, p. 96).

Mais ce style inquiet et agile, à travers ses bonds, laisse entrevoir un désarroi : la scène ne parvient pas à prendre corps. Les épisodes qui se succèdent n'ont pas de centre. "Je manque d'objectifs." ( $B$, p. 89) Le temps et l'espace ne se définissent qu'avec peine et pas assez: quelques noms de lieux déformés par les références cultivées (Monckton $[B, \mathrm{p} .14]$ ) ou fantaisistes (les Zétats-Zunis $[B$, p. 14], Monkeytown [ $B$, p. 73]), les vacances, les fins de semaine, les fêtes de famille. De là la nostalgie proustienne d'un "Temps" où s'édifierait le Livre, au sens de Mallarmé. 
Bloupe survit dans un monde sans géographie socialisable; ses cartes sont solipsistes et trompeuses, son almanach domestique. "J'sais pus où chus. Alors je mange." ( $B, p$. 122) La voracité est l'un des aspects rabelaisiens du roman, mais sans gaîté. Elle n'évite pas la tentation de l'exil: "Maybe I should go to some country also" ( $B, \mathrm{p} .126)$, phrase qui présuppose que le territoire habitable, le pays, est n'importe quel ailleurs.

On aurait tort de compter pour rien les évocations, souvent très suggestives, de l'habitat. Les maisons d'autrefois se délabrent. Des épaves jonchent les cours; toute une archéologie oubliée signifie pour rien l'éparpillement, la dispersion. On songe à la backyard de Guy Arsenault. Bloupe s'emploie lui aussi à construire une clôture, peut-être pour retenir un peu près de lui ce qui l'entoure. Au moins ses enfants! Mais le résultat? "un 2 x 4 dans l'œil * $(B$, p. 47). C'est ce que Tilleul racontera à Hilda le lendemain. Peut-être ne s'agit-il que de "bâti[r] quelque chose" $(B$, p. 50$)$, comme le pense le frère de Hilda. Le lieu domestique, le calendrier familial compensent-ils, dans l'intimité, l'absence de domicile reconnu?

Le projet littéraire, extrêmement ambitieux, se dit en termes modestes et matériels, dans une page admirable: l'intérieur d'une coque, d'une coquille, la nacre - ou le coq, voilà à quoi se réduit l'écriture, un refuge fragile, et une déjection animale: "piss clam philosophy"; oui: "les lettres se collent ensemble avant le Temps" ( $B, \mathrm{p} .55)$. La référence à Proust aboutit à l'auto-dérision: "Se perdre dans La recherche. Rien pour en faire une maison bien sûr." ( $B$, p. 56) Ainsi la littérature même devient inhabitable. Des "bribes" ( $B$, p. 68). Le coq et la moule laissent entendre un sens érotique, repris plus tard: l'alternance "bandénonbandé" $(B$, p. 70$)$. "Écrire ce qui sort et qui entre." ( $B$, p. 69) Va-et-vient, l'écriture ne progresse pas.

Ce roman ne se lit pas comme un roman. C'est du texte: brut, heurté, inventif, savant, espiègle, indécis, jamais chagrin mais vraiment mélancolique. Il témoigne pourtant d'une conscience littéraire acadienne: la plus désespérée, la plus fantasque et la plus cocasse. Le jeu des emprunts, des citations, des souvenirs, la multiplicité des tentatives, les esquisses de continuité, cette fois, récusent le génie moderne de l'organisation, de la progression, de la rationalité.

Que conclure? Il importe en premier lieu de souligner que la littérature acadienne d'aujourd'hui, tous genres confondus, et 
quelle que soit la qualité du texte envisagé, se préoccupe de l'espace habitable. Qu'elle essaie même de le constituer. Écrire, plutôt que s'installer devient un mode d'habitation. Étrange problème. Il suffit de songer à la littérature française: elle vit dans ses meubles, armoires de Guillevic, descriptions de Robbe-Grillet, discussions sur la décoration chez Nathalie Sarraute, choses et immeuble de Perec; en ce sens, elle demeure balzacienne: le personnage secrète autour de lui sa coquille, riche de significations. Processus naturel? Mais c'est ce que Bloupe ne parvient jamais à faire, ce que voudrait l'architecte de France Daigle, ce que n'envisage même pas Gérald Leblanc. Ces auteurs ne tiennent pas pour acquis ce qui se perd dans l'américanisation du monde, pudiquement nommée mondialisation: que les lieux soient individualisables, qu'on puisse y vivre dans une tranquille maîtrise, comme Dieu le septième jour. "Nowhere Now Here" $(B$, p. 22$)$, dit très bien Jean Babineau, qui rêve encore d'un "vrai matin de dieux grévistes" ( $B, \mathrm{p} .95)$.

À ce titre leurs livres offrent un recours: vivre du moins dans l'espace de la page, appropriable. Mais voici le plus surprenant, le plus instructif: au thème rebattu et immoral de l'identité, ces livres opposent celui de l'habitation, libre usage de l'espace. Or l'identité fige les peuples dans une représentation, mais surtout comme elle conçoit les hommes hors de leur action, dans l'authenticité prétendue de leur être, comme elle réduit leur histoire et leurs traditions à une image, elle leur prête sans qu'il en coûte rien une nature particulière et permanente: à condition de "respecter l'identité", tout sera permis aux grandes compagnies internationales. L'identité leur laisse le champ libre. Mais c'est notre champ!

L'architecture postmoderne l'a montré: une pierre de granit par-ci par-là dans le béton, et vous voilà en Bretagne! un gratteciel se termine par le faîte d'un buffet Henri II ou se pare de colonnes cannelées, et voilà du noble, beau comme l'antique! Comme on dit en France: cela fait genre, genre style, genre ambiance. La culture devenant un simple répertoire d'ornements, la liberté de l'ornementation est un attrape-nigaud insignifiant. Or habiter exige la maîtrise sémantique d'un espace: peupler un territoire, c'est un acte historique. Il est heureux que des écrivains s'en préoccupent. Leur habitat est le recueillement. "Contempler is the name of the game" ( $B$, p. 56), selon Bloupe, qui collectionne inlassablement ses feuilles, c'est le précipité de son 
46

existence; découvrir une contemporanéité qui inclue la distance, l'abolir en soi, c'est le projet de France Daigle; vivre dans l'instant poétique pour posséder sa ville, c'est l'œuvre de Gérald Leblanc. Le temps, plutôt que l'espace, établit notre demeure. 